\title{
Enimo-Energy Conservation System
}

\author{
M SriTeja, Ashfaq Nisar, K Vinay Kumar Reddy, G Vamshi Krishna
}

\begin{abstract}
In today's industrial production era, the usage of electricity is on the hike. All industrial machines use either electricity or solar energy. It is crucial for industrial appliances to work without any delays and interruption. To work without any delay, there should be scheduled maintenance from time-to-time and monitor the machine for any voltage hikes. The large-scale industries can afford a good \& expensive management system for the industrial machinery, but the small and medium scale industries cannot afford such an expensive system. Even, if they do possess an energy management system, the installed system is not up to the mark. To overcome this problem, we proposed a solution that helps in monitoring the performance of industrial machines, which can adapt to different environmental conditions, collect the data from the machines and process it. It not only monitors the appliances but also caters predictive information of appliance fault that can occur in the machine based on the data received. The most important thing is it is very inexpensive compared to other management systems in the market. The name of the proposed solution is "ENIMO" (Energy Information \& Monitoring). Enimo can be easily installed with the industrial appliances and be able to customize the dashboard to your liking.
\end{abstract}

Index Terms: Monitoring System, Data Analysis, Management System, Predictions, Cloud, Energy Wastage

\section{INTRODUCTION}

In today's era, the industries account for the major consumption of electrical energy in the country. In fact, $44 \%$ of the energy is used by industries in India. Due to the huge electricity demand, two major problems are being faced by the industry. One is the scarcity of energy and the other is escalated cost of the available energy. Industries have a great necessity for optimizing their energy needs and to decrease the cost for the same level of activity and increase the efficiency of the product in the industry.

To perform any production related task in the industry, electricity is required. Due to which, there is a constant rise in the requirement of electricity generation to meet the needs of an individual and professional. It is crucial in order to provide electricity continuously one must ensure that the consumption of electricity is not glut. The consumption of the electricity should be done in a productive manner. The major consumers of electricity in an industry are the industrial machines, electronic appliances, lighting and different electrical components in the industry. According to a study, if energy wastage is mitigated then $30 \%$ of energy consumption in the industry can be reduced.

Revised Version Manuscript Received on 16 September, 2019.

Makam SriTeja, Student B.Tech CSE, Vidya Jyothi Institute of Technology, Hyderabad, India makamsriteja@gmail.com

Ashfaq Nisar, Student B.Tech CSE, Vidya Jyothi Institute of Technology, Hyderabad, India ashfaqnisar00@gmail.com

K Vinay Kumar Reddy Student B.Tech CSE, Vidya Jyothi Institute of Technology, Hyderabad, India vinayreddy8579@gmail.com

G Vamshi Krishna Student B.Tech CSE, Vidya Jyothi Institute of Technology, Hyderabad, India vamshiginna1606@gmail.com

It is important that the electricity consumed by the machines should be constant during the runtime and there should not be any increase in the consumption of the energy. The energy consumption can be optimized by changing the environmental conditions of the machine, performing operations only when required and stopping the energy flow to the machine when it is not running.

According to [3]-[4], twenty percent of overall energy electricity consumption is due to industrial machinery and industrial buildings, which are increasing exponentially.

Enterprises today face many complications when it comes to energy consumption. They have a great need for an efficient consumption of energy in the industry. Industrial machinery consumes a lot of energy even when the machinery is in either standby or switched off mode. In addition, surplus energy consumption is due to the mechanical fault within the industrial machine. This fault causes an excess consumption in the energy during the runtime of the machine.

In addition, one of the notable problems is not only finding out the fault in the machine but also the energy wastage caused by the machine. In the large-scale industries, the consumption of energy is merely not important but stopping the machines from any breakdown is very important.

The industries aim to achieve a large number of goods/products in a less span of time, which in turn leads the machine to produce more goods and decreases the efficiency of the machine. The efficiency of the machine is mainly decreased when it is overloaded with energy.

In this paper, the proposed solution Enimo will not only monitor the energy consumption but also analyze patterns of the current running machine. The data collected from the machine is sent to the cloud to help predict the next maintenance and breakdown of the machine. There is a main hub, which uses the IOT technology to gather the data from all the sensors and machines.

\section{RELATED WORK}

In the past, many systems have been built for the industries to monitor the energy consumption of machines. For example, in [7], the author designed a monitoring system for machinery using Acme. It is an alternate current power meter, which gives the energy consumption details through a web interface. Similarly, in [6] the author represents the same idea as that in reference [7] but instead of monitoring the consumption it provides details about how, where and when the energy is passed to a machine. However, similar power-conservation systems were developed as described in [8]-[9]. There are systems in the market, which can even control appliances according to the power usage of appliance as described by the author in [9]. In which, the system automatically adjusts itself to the environmental conditions. 
Therefore, as per the above-mentioned references. Many systems have been developed for the energy monitoring. The solution proposed in this paper will not only emphasize on monitoring of machinery. However, it helps in predicting the condition of the machine in the future. Based on the voltage data gathered, we can also predict the faults in the machine.

\section{PROPOSED SOLUTION}

The Enimo does energy consumption monitoring of machines in industries using IOT in real time. As the data is being received from the machines, we process them in the cloud and display the real-time insights to the user and generate the predictions on the collected data by using LSTM machine learning algorithms and data analysis to empower the decision making of an organization.

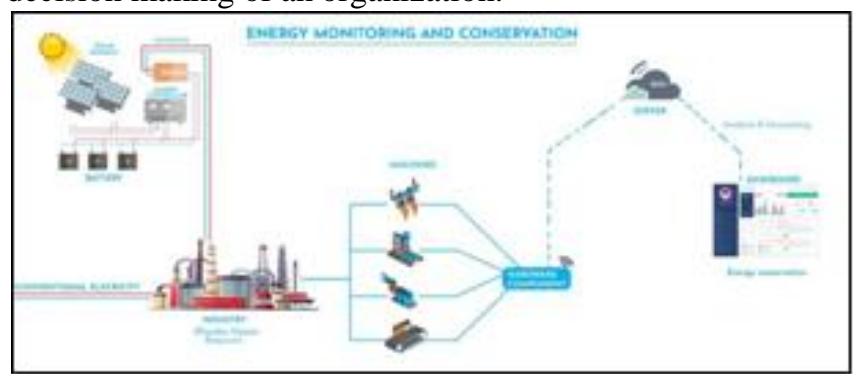

Fig 1. Workflow Diagram

Enimo has three main objectives to fulfill and they are:

1. Identify faults and weaknesses in the machine early so preventative maintenance and replacement of the components can be done in a safe and orderly way

2. Ensure the machine is maintained at an optimum environment to achieve maximized performance and life.

3. Achieve energy efficiency by providing the required minimal energy to the machine.

Working: ENIMO hardware's control hub is installed at the input power supply of the machine and main source of the energy. The INA219 sensor is used to collect the current and voltage values consumed. The INA219 sensor will read the real-time readings of the machine's current and voltage values.

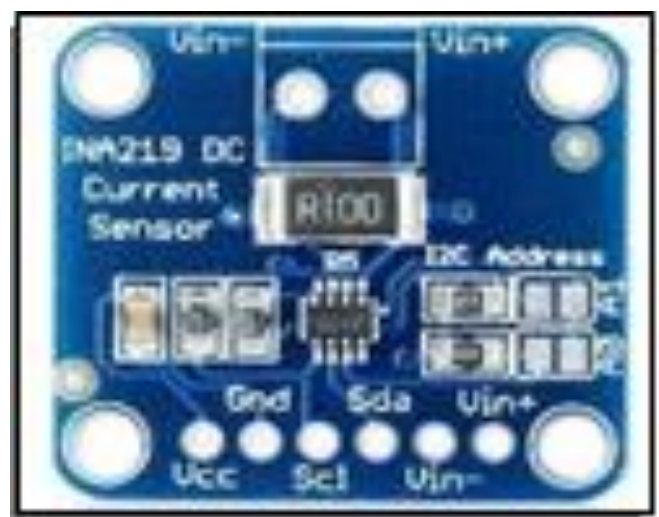

Fig 2. INA219 sensor

These valuable readings are sent to control hub. The control hub sends the data to the cloud for processing.

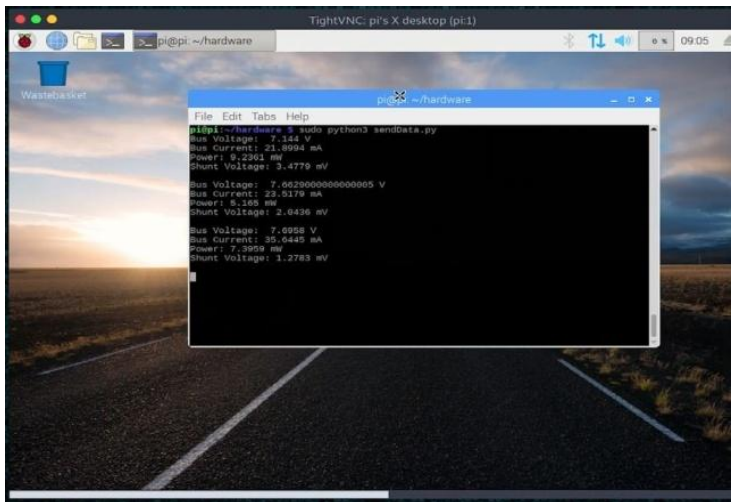

Fig 3. Real-time readings from the sensor

These values are displayed on the React dashboard which provides a better visual aid to the user to track each necessary piece of information and take the right decision at the right time. ENIMO is designed to capture the power and voltage signatures for every 20 seconds. It means we have 4320 readings per each day, 30240 per week and 129600 per month. Now we have all sorts of points, it's almost like too much of the information.

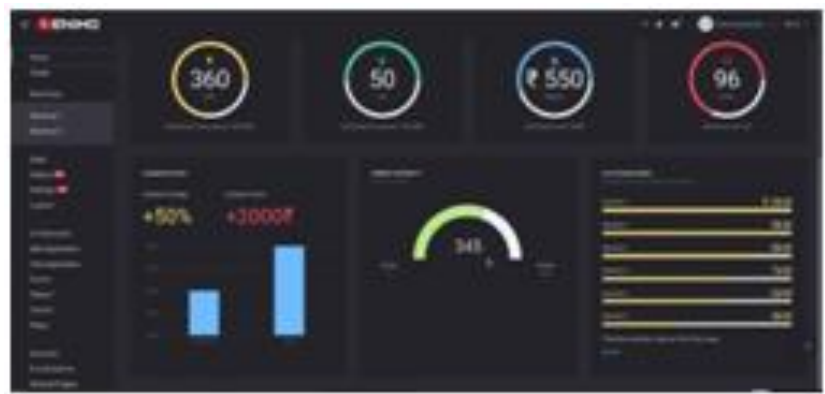

Figure 4. Insights displayed on the dashboard

This huge data can be interpreted and used in generating predictions for decision-making. Coming to data analysis, the data stored in the database is first cleaned to reduce noise in the data. After smoothening the data, exploratory data analysis is made to gain more domain knowledge and check what parameters are influencing the output that is energy consumption by finding root mean square values. We use a regression model to generate the prediction. As we are dealing with machinery, from the available data, $80 \%$ of data is used for training and $20 \%$ of data is used for the testing. Alerts generated are sent to the user through SMS (using MSG91 SMS gateway), Email and notification will be displayed on the dashboard

Our solution targets:

Small and medium scale industries, factories, distribution facilities where there is a lot of machinery.

Why Enimo?

Comparing electricity consumption and bills: ENIMO provide us with a feature of comparing the power consumption and power bills to find the reasons for fluctuations in the power bills and aims to achieve optimal power consumption.

Find out key energy consumers: Spots the hidden energy consumers in the industry and notifies the user.

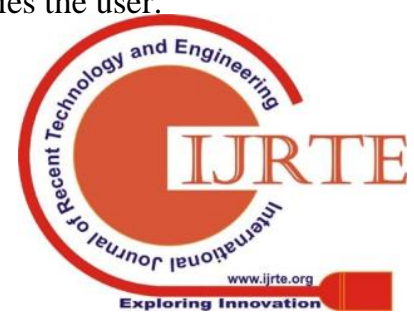


Set the monthly budgets: You can set a monthly budget for your power bill and ENIMO will constantly keep track of the consumption and alerts the user if the estimated bill goes beyond the set budget.

Diagnose specific area of wastage of energy: Save time by having remote access to machine data and pinpointing faults to specific individual machines.

Draw energy consumption trends: Displays the real-time insights with a better visual aid. User can view daily, weekly and monthly insights.

Cost efficient: This project is pocket-friendly and avoid costly downtime and protect your business from losses.

Managing energy consumption: This project focuses on managing energy consumption rather than accepting it as a fixed cost and cannot be controlled.

\section{Technologies used:}

ReactJS - We have used ReactJS for this project because when there are any new values in the database then the whole page will be updated if we use normal web technologies but in the case of the ReactJS only the single component will be updated whose value is changed. As per [10]-[11] references ReactJS has been used.

Bootstrap-We used bootstrap to make the website responsive even if the user opens the web page in his phone, tablet or laptop, the website will automatically adjust itself to the size of the screen.[12]-[13] has been referred to develop.

Python-Python is mostly used in hardware to send the data to the server which is fetched from the sensors.

Firebase - At the backend, we have used firebase as the database because all the connections/requests made will be secure. The read/write speeds of the database are very fast compared to any other database.[14]-[15] has been referred for using firebase.

Google Cloud - The Google Cloud comes into the picture to analyze the data that we receive from the firebase and send the results back to the firebase. So, the ENIMO dashboard will be able to fetch the values from the firebase.[16]

Data Analysis - After fetching the data from the firebase, we wrote an algorithm in google cloud which will keep on taking the data from the firebase and executing different algorithms on the data.

IOT-As Computer Science Students, we have not learned the IOT anywhere but for this challenge, we gained knowledge about the IOT mostly about the different sensors and programming these sensors.

\section{IV.EXPERIMENT RESULTS}

To conduct an Experiment first Enimo Monitor is connected directly into the breaker panel. It is the brains of the operation. It collects and sends data through your home's/Industry's Wi-Fi so you can track machines energy usage from anywhere on your smartphone or computer. A qualified electrician can install the ENIMO monitor in your electrical panel without any rewiring, and usually in less than 30 minutes. The Monitor consists of sensors, or current transformers, clamp around the main power leads feeding your Industry. There is also an antenna for connecting to $\mathrm{Wi}-\mathrm{Fi}$. This gives you real-time data on energy consumption and production, allowing you to Make smarter decisions about energy use, recognize abnormal patterns of energy use, pointing to potential problems of various appliances, estimate the energy bill, allowing you to see the impact of your energy use on your wallet in real time

During the first 24 hours after installation, the ENIMO monitor goes through an automated signal check and calibration process. As soon as that process is complete, you can start learning about your energy use right away, with Enimo's real-time metering which is displayed in the dashboard.

The access to ENIMO dashboard is end-to-end authenticated. The user based on subscription gets an email and password. When a user has login, he can see the visual insights on the dashboard. Consisting of various machines data and attributes like Total Energy consumption of machines which tells the amount of energy consumed by all the machines

While you are doing that, the latest trends in machine learning algorithms i.e LSTM models will be working in the background to identify the electrical signatures of specific machines within your industry, so you can see how much energy they are using and this information is displayed on the dashboard.

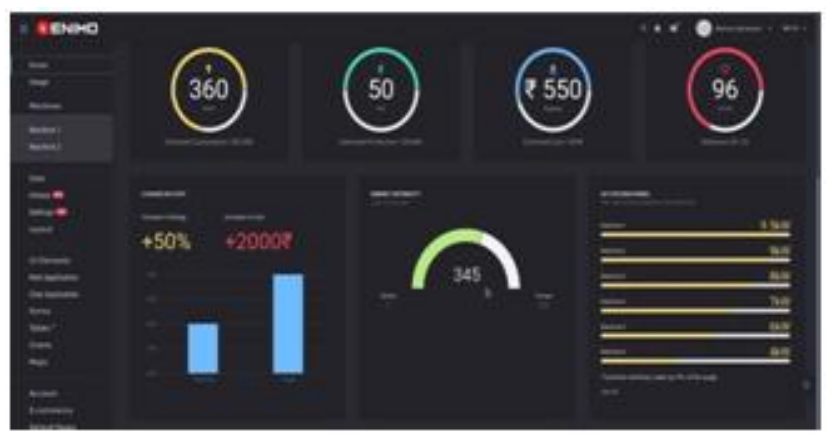

Figure 5. Page of Dashboard

The figure [5] displays home page of the dashboard, which shows power consumption details of individual machines and also all the machines. Our Dashboard also displays estimated power bill based on power consumed using predefined background calculations and predicts the future usage and respective power cost.

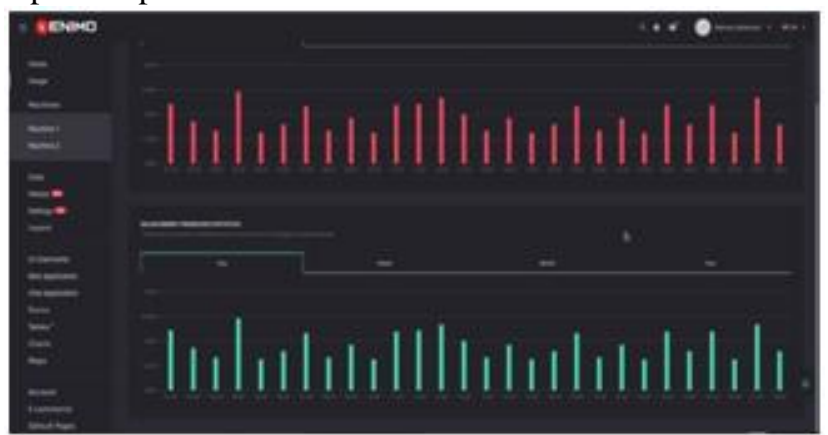

Figure 6. Dashboard displaying monthly wise insights

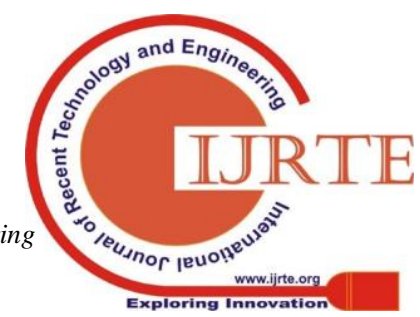


We are even working on ways that Enimo monitor can help identify technical problems in machines that might be malfunctioning, so you can get a heads up before your machine quits, or before an electrical issue becomes dangerous. In dashboard as shown in [6] for a set of machines if it shows, red color it means the machine is consuming more than the required amount: which indicates inefficiency of a machine and the user can go for maintenance beforehand this is known as Predictive maintenance. ENIMO also helps in making better decisions.

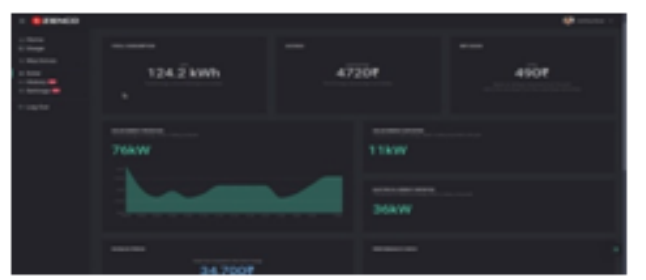

Figure 7. Dashboard displaying Stats and Usage

The Figure [7] shows Machine profile page that has a list of all the machines available in the industry. A user can view energy consumption details of each machine. Energy vs time bar graph is plotted to display energy consumption details of an individual machine, daily wise to the yearly wise making user more aware of the utilization and performance of the machine. This report progress identifies changes in the consumption and make suggestions that are specific and tailored to your machines to help you cut costs the following week.

ENIMO stores information of all machines for better predictions by understanding the patterns.

In addition to this machine page also provides some other details about a machine which includes machine status, total working hours, total energy consumed with the respective cost for units consumed. This page lets you find out the key energy consumers of an organization and the time when it consumed more than the required energy.

Based on total energy consumption bill is calculated. Moreover, it shows the machines in the Industry with the amount of Energy consumed by Individual Machine.

\section{CONCLUSION}

ENIMO is an energy intelligence system. It acts like an interactive energy efficiency solution for entire Industry either it is Micro, Small or Medium, which consists of sensors that connect directly to your breaker box. ENIMO monitors every machine that uses electricity. Enimo processes your electrical usage data and shares insights to help you make your machines smarter, more energy efficient and safer.

It also helps people to avoid blindly paying off electricity bill without knowing where that money is going. As most industries waste more than $30 \%$ of the electricity, they pay for. ENIMO uncovers energy trends, pinpoints, power hogs, and gives you control over your machines and your electricity bill. Enimo reduces your energy use with device level notifications and you can set goals to track your progress and stay on budget.

Thus, ENIMO web interface dashboard show you what's driving the cost of your electricity bill, share energy saving tips, and send electricity safety alerts. With ENIMO, you can power your machines smarter.

\section{FUTURE SCOPE}

As of now, Industries are looking for alternative sources of energy as energy from the government has escalated prices per unit. The most preferable alternative renewable resource is Solar Energy as we in India are having a huge amount of sun's energy available around the year.

In Future, we also tend to provide another interesting feature (i.e. Monitoring Your Solar Plant). Using this feature, you can monitor everything going on inside your industry as well as your solar Plant and see how much energy is generating compared to how much grid electricity you are using in real time and for less money than traditional solar monitoring systems. It also shows the estimate payback period based on energy generation and usage and can even detect if there is something wrong with your solar plant and let you know it in right away.

\section{REFERENCES}

1. International Energy Agency "Cool Appliances. Policy strategies for energy efficient homes", Paris, France, 2003.

2. O. Gassmann, H. Meixner, J. W. G. J. Hesse, and W. Gopel, Sensors in Intelligent Buildings: Sensors Applications. Wiley-VCH, 2001 .

3. 2011, 11th International Conference on Intelligent Systems Design and Applications. An Intelligent System for Electrical Energy Management in Buildings .Giuseppe Anastasi, Francesco Corucci, Francesco Marcelloni.

4. Energy Information Administration," International Energy Outlook 2010- Highlights “. www.eia.doe.gov/oiaf/ieo/highlights.html.

5. www.eia.doe.gov/emeu/cbecs, "Commercial Buildings Energy consumption Survey"2003. USA DOE Energy Information Administration.

6. X. Jiang, S. Dawson-Haggerty, P. Dutta, D.Culler, 'Design and implementation of a high-Fidelity AC metering network'. Proc. ACM/IEEE Conference on Information Processing in Sensor Networks (IPSN 2009), San Francisco, USA, 2009.

7. X. Jiang, M. Van Ly, J. Taneja, P. Dutta, D.Culler, 'Experiences with a high-Fidelity Wireless Building Energy Auditing Network', Proc. ACM SenSys 2009, Berkeley, USA, 2009.

8. S. Helal, W. M. H. E. Zabadani, J. King, Y. Kaddoura, and E. Jansen, "The gator tech smart house: a programmable pervasive space," IEEE Computer, vol. 38, no. 3, pp. 50-60, 2005.

9. Ruzzelle, A. Schoofs, G. O'Hare, "Real-time appliances Recognition and Profiling Through A single energy monitoring sensor, Proc IEEE conference on Sensor, Mesh and Ad Hoc Communication and Networks" Boston, USA, 2010.

10. "React.js Essentials", Published by Packt Publishing Ltd. Artemij Fedosejev.

11. 'REACTJS BLUEPRINTS' BY SVEN A.ROBBESTAD

12. 'An introduction to the bootstrap', B Efron, RJ Tibshiran

13. "Bootstrap methods and their application", by AC Davison, DV Hinkley - 1997 books.google.com

14. Firebase. By F Cheng - 2018 - Springer.

15. "The Definitive guide to firebase: build Android apps on Google's mobile platform". L Moroney - 2017 - books.google.com.

16. “Cloud computing”, N Antonopoulos, L Gillam - 2010 - springer. 


\section{AUTHORS PROFILE}

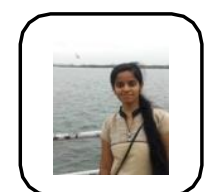

Makam SriTeja is a student pursuing Bachelors in Computer Science and Engineering in Vidya Jyothi Institute of Technology, Hyderabad, India. Her interests are in Internet of Things Applications, Data Science and Machine Learning.

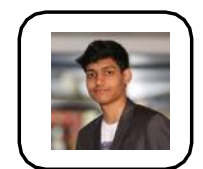

Ashfaq Nisar is a student pursuing Bachelors in Computer Science and Engineering in Vidya Jyothi Institute of Technology, Hyderabad, India. His interests are in Data Science and Machine Learning.

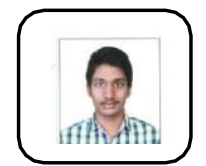

K Vinay Kumar Reddy is a student pursuing Bachelor in Computer Science and Engineering in Vidya Jyoth Institute of Technology, Hyderabad, India. His interests are in Data Science and Machine Learning.

G Vamshi Krishna is a student pursuing Bachelors in Computer Science and Engineering in Vidya Jyothi Institute of Technology, Hyderabad, India. His interests are in Data Science, Machine Learning. 